\title{
Preliminary study on the diagnostic value of cardiac magnetic resonance feature tracking for malignant ventricular arrhythmias in non-ischemic dilated cardiomyopathy
}

\author{
Linsheng Song ${ }^{1 \#}$, Xinyi Zhao" ${ }^{1 \#}$, Wenlong Lv $^{2 \#}$, Jie Zeng ${ }^{3}$, Yishuang Wang ${ }^{1}$, Bo Gong ${ }^{4}$, \\ Andreas P. Kalogeropoulos ${ }^{5}$, Hong Pu ${ }^{1 *}$, Yifeng Bai ${ }^{6 *}$, Shengkun Peng ${ }^{1 *}$
}

${ }^{1}$ Department of Radiology, Sichuan Academy of Medical Sciences and Sichuan Provincial People's Hospital, University of Electronic Science and Technology of China, Chengdu, China; ${ }^{2}$ Department of Radiotherapy, Cancer Center, The First Affiliated Hospital of Fujian Medical University, Fuzhou, China; ${ }^{3}$ Department of Cardiology, Sichuan Academy of Medical Sciences \& Sichuan Provincial People's Hospital, University of Electronic Science and Technology of China, Chengdu, China; ${ }^{4}$ Human Disease Genes Key Laboratory of Sichuan Province and Institute of Laboratory Medicine, Sichuan Academy of Medical Sciences \& Sichuan Provincial People's Hospital, University of Electronic Science and Technology of China, Chengdu, China; ${ }^{5}$ Division of Cardiology, Department of Medicine, Stony Brook University Renaissance School of Medicine, Stony Brook, New York, NY, USA; 'Department of Oncology, Sichuan Provincial People's Hospital, University of Electronic Science and Technology of China, Chengdu, China

Contributions: (I) Conception and design: S Peng, Y Bai, L Song; (II) Administrative support: H Pu, S Peng, Y Bai, B Gong; (III) Provision of study materials or patients: S Peng, Y Bai, B Gong, J Zeng; (IV) Collection and assembly of data: L Song, X Zhao, Y Wang; (V) Data analysis and interpretation: L Song, X Zhao, S Peng, Y Bai; (VI) Manuscript writing: All authors; (VII) Final approval of manuscript: All authors.

\#These authors contributed equally to this work.

*These authors contributed equally to this work.

Correspondence to: Shengkun Peng, MD. Department of Radiology, Sichuan Academy of Medical Sciences and Sichuan Provincial People's Hospital, University of Electronic Science and Technology of China, Chengdu, China. Email: pengsk1986@126.com; Yifeng Bai, MD. Department of Oncology, Sichuan Provincial People's Hospital, University of Electronic Science and Technology of China, Chengdu, China, Chengdu, China. Email: 15756285949@163.com; Hong Pu, MD. Department of Radiology, Sichuan Academy of Medical Sciences and Sichuan Provincial People's Hospital, University of Electronic Science and Technology of China, Chengdu, China. Email: ph196797@163.com.

Background: Patients with nonischemic dilated cardiomyopathy (NIDCM) and malignant ventricular arrhythmia (MVA) often have a poor prognosis and a high risk of sudden cardiac death. Although the diagnosis of MVA is straightforward by electrocardiogram (ECG), the underlying abnormalities of ventricular mechanics in these patients are unknown. This study aims to preliminarily explore the value of cardiac magnetic resonance feature tracking (CMR-FT) for MVA in dilated cardiomyopathy.

Methods: In this retrospective study, patients with NIDCM who met inclusion criteria were divided into an MVA group and a non-MVA group (included from January 2018 to September 2021). The interobserver agreement of myocardial strain parameters, including global longitudinal strain (GLS), global circumferential strain (GCS) and global radial strain (GRS), were tested. The GLS, GCS, GRS, left ventricular ejection fraction (LVEF), $\mathrm{T}_{\text {peak }}-\mathrm{T}_{\text {end }}$ interval on ECG and brain natriuretic peptide (BNP) were compared between groups. Single-factor and multifactor receiver operating characteristic (ROC) curve analyses were conducted to calculate the area under the ROC curve (AUC), cut-off point, sensitivity, and specificity of these parameters in predicting MVA in NIDCM.

Results: A total of 161 NIDCM patients were included (54 in the MVA group). GLS, GCS, and GRS had good interobserver agreement (all intraclass correlation coefficients $>0.80$ ). The absolute GLS and GCS, GRS and LVEF were lower in the MVA group than the non-MVA group $(\mathrm{P}<0.001), \mathrm{T}_{\text {peak }}-\mathrm{T}_{\text {end }}$ and BNP were higher $(\mathrm{P}<0.001)$. Single-factor ROC curve analysis showed that GLS, GCS and GRS had certain diagnostic value for MVA (AUC $=0.795,0.802$, and 0.754 , respectively). Among them, GCS had higher sensitivity and specificity (GCS 0.796/0.776, GLS 0.778/0.757, GRS 0.741/0.692). Multifactor ROC curve analysis showed the combination of GLS and GCS (AUC $=0.810$ ), the combination of GCS and GRS (AUC $=0.802$ ), the 
combination of GLS and GRS (AUC =0.787), the combination of GLS, GCS, and GRS (AUC $=0.810$ ).

Conclusions: The three-dimensional myocardial strain parameters (especially GLS and GCS) measured by CMR-FT had certain diagnostic value and could reflect the underlying abnormality of ventricular mechanics of NIDCM with MVA.

Keywords: Dilated cardiomyopathy; malignant arrhythmia; ventricular strain; tracking

Submitted Nov 22, 2021. Accepted for publication Feb 22, 2022.

doi: $10.21037 /$ atm-22-660

View this article at: https://dx.doi.org/10.21037/atm-22-660

\section{Introduction}

Nonischemic dilated cardiomyopathy (NIDCM) is a myocardial disease characterized by systolic dysfunction without abnormal load or significant coronary artery disease but with left ventricular or biventricular dilatation. NIDCM patients with malignant ventricular arrhythmia (MVA) have a higher risk of sudden death (1). Therefore, accurate prediction of MVA has clinical value. Brain natriuretic peptide (BNP) is an independent predictor of rapid ventricular arrhythmia (2), and the $\mathrm{T}_{\text {peak }}-\mathrm{T}_{\text {end }}$ interval $\left(\mathrm{T}_{\mathrm{p}}-\right.$ $\mathrm{T}_{\mathrm{e}}$ ) on the electrocardiogram (ECG) has high specificity and sensitivity for predicting MVA (3). Although the diagnosis of MVA is straightforward through ECG, the underlying abnormalities of ventricular mechanics in these patients are unknown. The final outcome of NIDCM with MVA is heart failure, or even sudden death, so it is very important to evaluate the heart function. However, past studies have focused on ECG and biomarkers, while there are relatively few relevant imaging studies.

NIDCM is characterized by myocardial fibrosis and ventricular remodeling, which not only are an important pathophysiological basis of arrhythmia but also lead to abnormal myocardial systolic and diastolic function. Myocardial fibrosis quantitatively assessed by late gadolinium enhancement (LGE) and T1-mapping is a powerful predictor of ventricular arrhythmia $(4,5)$, but there is relatively little research on myocardial strain in this context. At present, cardiac magnetic resonance tagging is considered the gold standard for myocardial strain measurement (6), but it needs to prospectively obtain marker images in the scan to be analyzed, so its temporal resolution is limited. Recently, a new cardiac magnetic resonance feature-tracking (CMR-FT) technique based on conventional magnetic resonance cine sequences has attracted the attention of scholars. CMR-FT not only has the advantages of simple operation, high image spatial resolution, and no need to add special sequences, but it can quickly postprocess the images to obtain the values of myocardial strain parameters. It can also detect subtle changes in cardiac function before ejection fraction changes $(7,8)$. In addition, compared with two-dimensional CMRFT, three-dimensional (3D) CMR-FT offers superior repeatability and variability within and between observers $(9,10)$. The myocardial strain parameters measured by 3D CMR-FT are important for accurately evaluating myocardial strain and can predict left ventricular reverse remodeling in patients with severe NIDCM (11). Therefore, this study aims to preliminarily explore the value of cardiac magnetic resonance feature tracking (CMR-FT) for MVA in dilated cardiomyopathy.

We present the following article in accordance with the STARD reporting checklist (available at https://atm. amegroups.com/article/view/10.21037/atm-22-660/rc).

\section{Methods}

\section{Patient population}

Patients with dilated cardiomyopathy hospitalized in the Cardiology Department of the Sichuan Provincial People's Hospital from January 2018 to September 2021 were retrospectively included in the study according to the diagnostic criteria (12) of the Classification and Definition Criteria of Cardiomyopathy published by the American Heart Association in 2006. All procedures performed in this study involving human participants were in accordance with the Declaration of Helsinki (as revised in 2013). The study was approved by the Institutional Ethics Review Committee of the Sichuan Provincial People's Hospital (No. 538). Individual consent for this retrospective analysis was waived.

Inclusion criteria: patients with MR image quality 
that met the diagnostic requirements; with a diagnosis of dilated cardiomyopathy by clinical and imaging methods and confirmed to have no obvious abnormality of the coronary artery by coronary angiography or CT coronary angiography; and a 24-hour dynamic ECG (Holter), inpatient ECG, and BNP within 2 days before and after cardiac magnetic resonance examination. Exclusion criteria were: valvular heart diseases; electrolyte disturbance, left ventricular hypertrophy, atrial fibrillation, bundle branch block, pre-excitation syndrome, or a pacemaker; antimalignant arrhythmia treatment was performed during the patient's study period; incomplete clinical data. All patients were divided into two groups according to the occurrence of ventricular arrhythmia, namely, an MVA group (dilated cardiomyopathy with MVA) and a non-MVA group (dilated cardiomyopathy without MVA).

\section{ECG and image analysis}

MVA defined as Clinical presented as hemodynamic disorders, ECG showed malignant premature ventricular rhythm, paroxysmal ventricular tachycardia, persistent ventricular tachycardia, torsive ventricular tachycardia, ventricular flutter and ventricular fibrillation.

The ECG analyses were performed by two experienced cardiologists who were fully blinded to the clinical outcomes and CMR results. If there was discrepancy between the two reviewers, a third senior reviewer was invited to make the final judgment. ECG analyses combine 24-hour dynamic ECG (Holter) with inpatient ECG.

For the measurement of the $T_{p}-T_{e}$ interval, each ECG was magnified 10 times to measure the $T_{p}-T_{e}$ interval from the ECG database. $T_{p}$ refers to the highest peak of the $T$ wave. If the T-wave was bimodal, the higher peak was taken as $T_{p} . T_{e}$ refers to the intersection of the descending branch of the $T$ wave and the baseline. If there was a $U$ wave, the notch between the $T$ wave and the $U$ wave was taken as $T_{e}$. The $T_{p}-T_{e}$ interval was measured by two experienced ECG doctors. The precordial leads V2-V4 were selected as the unified choice of $T_{p}-T_{e}$ interval measurement in each case, and the mean values of three heartbeat calculation data were measured in each lead.

\section{MRI technique and image analysis}

All patients underwent MRI examination at $1.5 \mathrm{~T}$ (Magnetom Aera, Siemens) with an six-channel coil. All cine images were acquired with 20-25 phases per cardiac cycle. Cardiac short- axis and two-, three-, and four-chamber view cine images were acquired using a standard breath-held steady-state free precession cine sequence. Typical imaging parameters were as follows: section thickness, $8 \mathrm{~mm}$; gap, $2 \mathrm{~mm}$; repetition time, 3.0-3.4 msec; echo time, $1.5-1.7$ msec; matrix size, $192 \times 224$ to $224 \times 256$; field of view, $320 \times 320$ to $380 \times 380 \mathrm{~mm}^{2}$; and temporal resolution, $30-55 \mathrm{msec}$, depending on heart rate (13).

MRI analyses were performed by two experienced radiologists who were fully blinded to the clinical outcomes and ECG results. Strain analysis at cardiovascular MRI was performed using the QStrain package (Medis Medical Imaging Systems). In end-systole and end-diastole, the endocardial and epicardial contours were automatically detected with manual correction. Papillary muscles were excluded from the endocardial contour (14). Then, three-directional 3D myocardial strain parameters were derived: global radial strain (GRS), global circumferential strain (GCS), and global longitudinal strain (GLS). Radial and circumferential strain parameters were derived from the short-axis stack, whereas longitudinal strain parameters were derived from the two-, three-, and four-chamber views (Figure 1).

\section{Statistical analysis}

The normality test and homogeneity of variances test were performed on the quantitative data. According to the test results, the two-independent-samples-t-test or the MannWhitney $\mathrm{U}$ test was run to compare a variable between groups. All measured values are expressed as mean \pm standard deviation or median (interquartile range). Singlefactor and multifactor receiver operating characteristic (ROC) curve analyses were used to calculate the AUC, cutoff point, sensitivity, and specificity of myocardial strain parameters (GLS, GCS, and GRS), $T_{\mathrm{p}}-\mathrm{T}_{\mathrm{e}}$ interval, and BNP (AUC, specificity and sensitivity $>0.50$, good). The level of agreement between reviewers was assessed using intraclass correlation coefficients (ICCs), ICCs are reported with $95 \%$ their confidence intervals (CIs). $\mathrm{P}<0.05$ was considered statistically significant, $\mathrm{P}<0.05$ was two-sided. SPSS 22.0 software was used for statistical analysis.

\section{Results}

\section{Clinical characteristics}

A total of 161 NIDCM patients were included in this study (Figure 2), including 84 females and 77 males, with an average age of 55 years. There were 54 patients in the MVA 

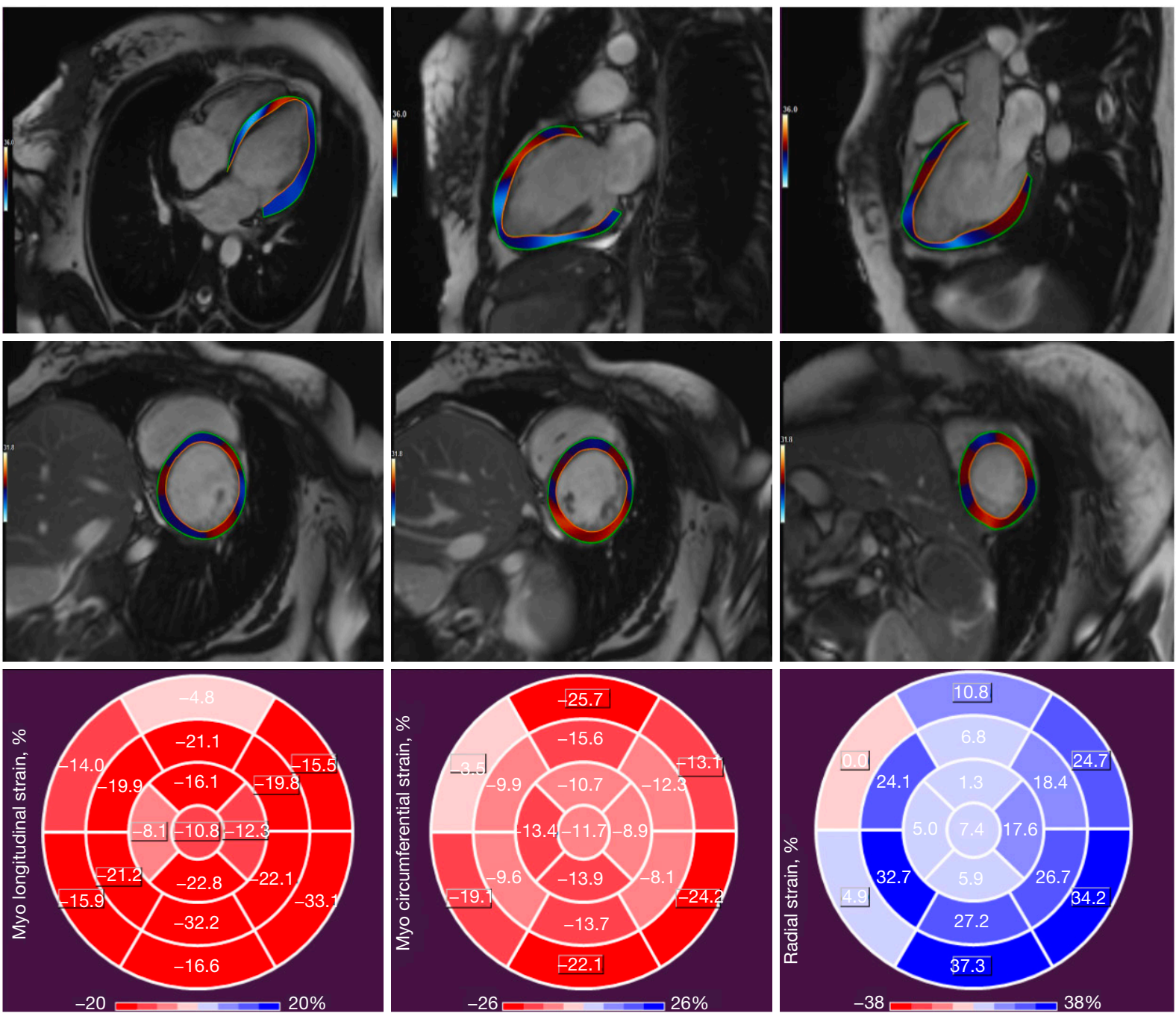

Figure 1 Feature-tracking analysis was performed on routine cardiac cine images (short-axis views and two-, three-, and four-chamber views). In long-axis and short-axis views, the endocardial and epicardial contours were automatically detected with manual correction. The deeper orange and blue indicate poor heart function. MRI, magnetic resonance imaging; Myo, myocardial.

group and 107 patients in the non-MVA group. There was no significant difference in patient characteristics (age, sex, weight, body mass index) between the two groups ( $\mathrm{P}>0.05)$, but the heart rate (beat $/ \mathrm{min}$ ) in the MVA group was significantly faster $(108 \pm 11$ vs. $76 \pm 13, \mathrm{P}<0.001)$ than that in the non-MVA group. The baseline population characteristics are listed in Table 1.

\section{Study outcomes}

\section{Interobserver agreement}

Two radiologists independently performed strain postprocessing, and the measured values were analyzed by ICCs. The results showed that all the left ventricular global 3D strain parameters had ICC $>0.80$ (Table 2).

\section{Ventricular mechanics}

GLS, GCS, $T_{p}-T_{e}$, and BNP were significantly higher in the MVA group than in the non-MVA group $(\mathrm{P}<0.001)$, and the GRS and left ventricular ejection fraction (LVEF) were significantly lower $(\mathrm{P}<0.001)$ (Table 3).

\section{Prognostic performance}

ROC curves of myocardial strain parameters (GLS, GCS, 


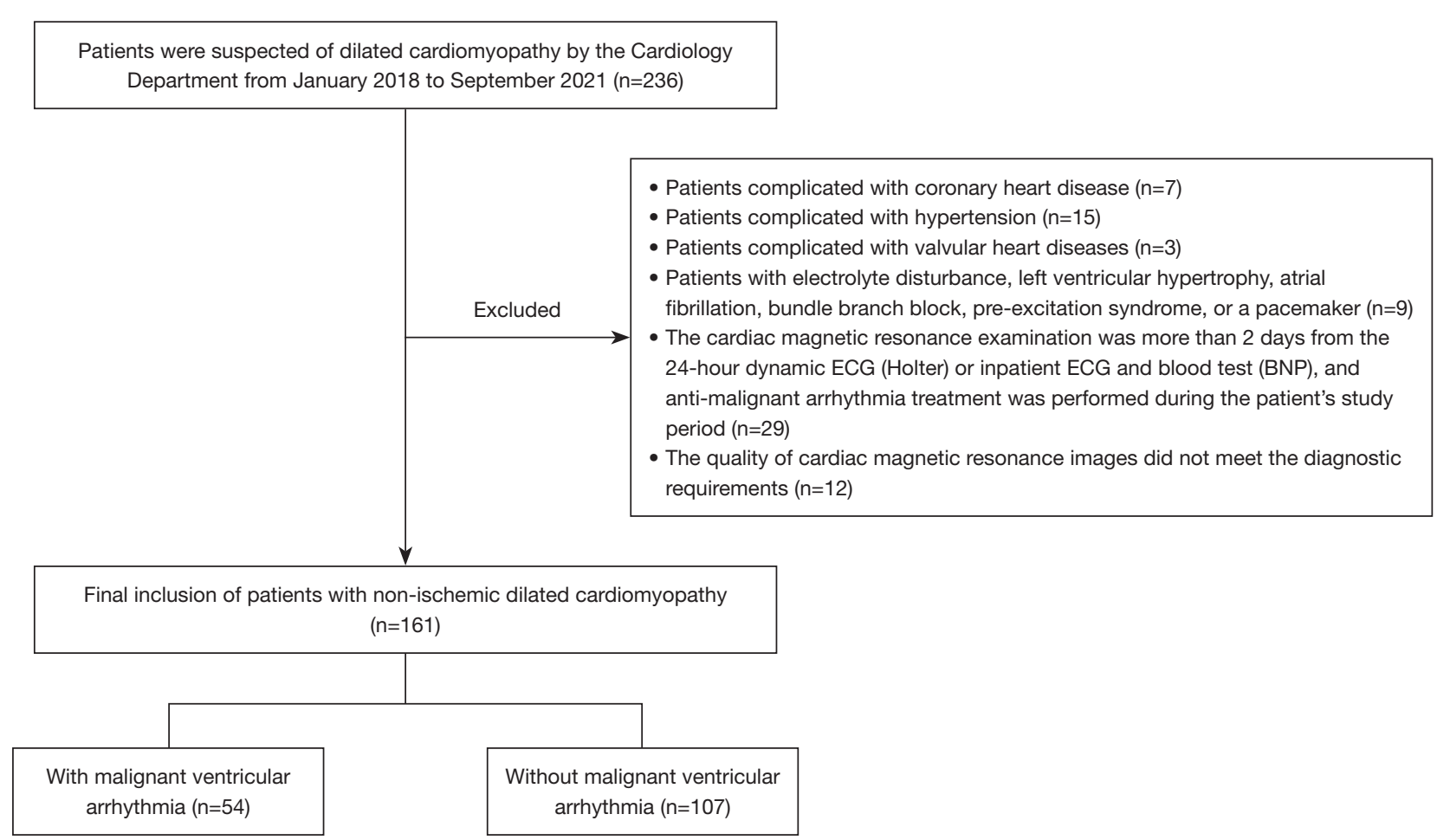

Figure 2 Patient flowchart. ECG, electrocardiogram; BNP, brain natriuretic peptide.

Table 1 Clinical characteristics of all patients

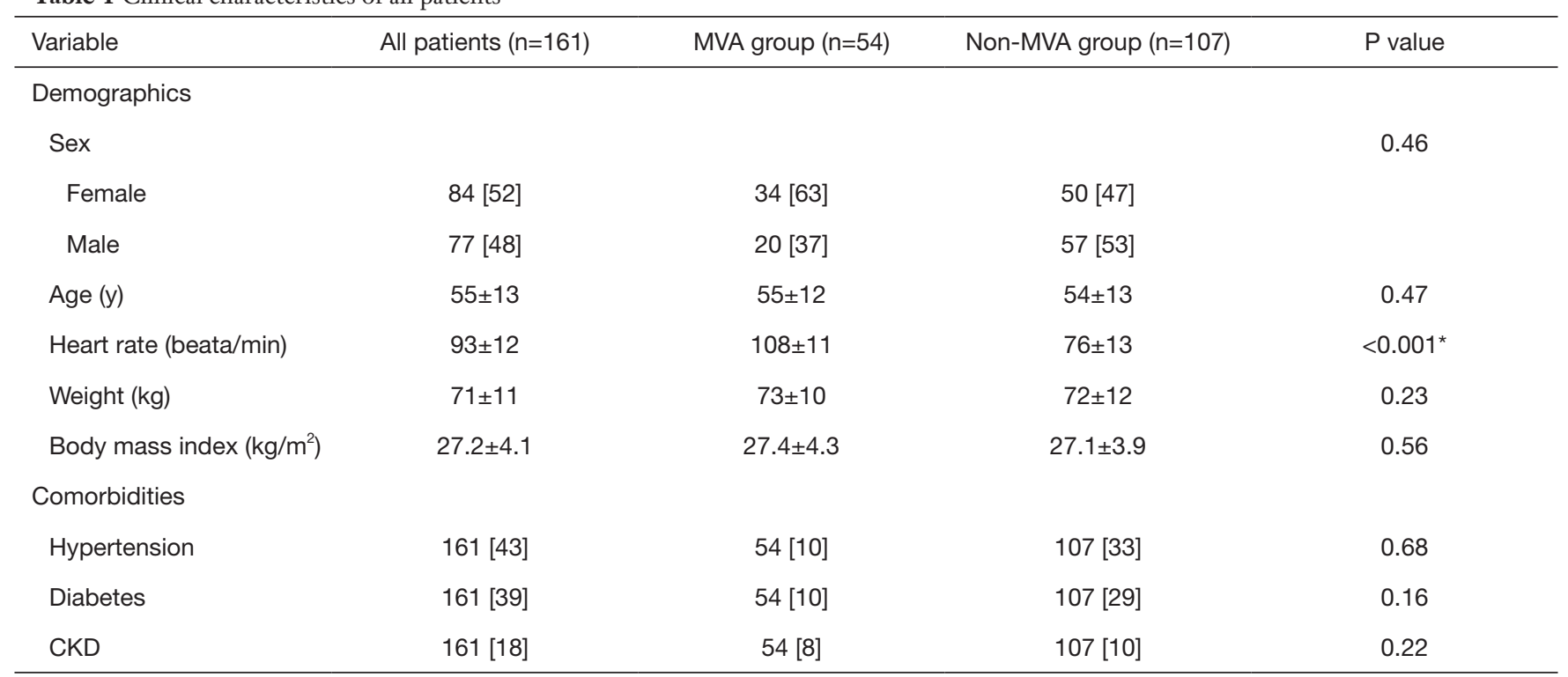

Data are displayed as mean \pm standard deviation or frequency [\%]. *, statistically significant difference. MVA, malignant ventricular arrhythmia; CKD, chronic kidney disease. 
Table 2 Intraclass correlation coefficient of CMR-FT 3D strain parameters in the NIDCM group

\begin{tabular}{lll}
\hline Variable & ICC & ICC 95\% Cl \\
\hline GLS & 0.938 & $0.745-0.985$ \\
GCS & 0.925 & $0.741-0.982$ \\
GRS & 0.942 & $0.758-0.986$ \\
\hline
\end{tabular}

ICC, intraclass correlation coefficient; $\mathrm{Cl}$, confidence interval; GLS, global longitudinal strain; GCS, lobal circumferential strain; GRS, global radial strain; NIDCM, nonischemic dilated cardiomyopathy; CMR-FT, cardiac magnetic resonance feature tracking. and GRS), LVEF, $\mathrm{T}_{\mathrm{p}}-\mathrm{T}_{\mathrm{e}}$ interval, and $\mathrm{BNP}$ were drawn for MVA (Table 4 and Figure $3 A$ ).

In the multifactor ROC analysis, pairwise combinations of the three myocardial strain parameters and the combination of all three myocardial strain parameters were input as independent variables $(\mathrm{X})$, and the prediction probability $\mathrm{P}$ based on each combination of factors was calculated by binary logistic regression. The results showed that GLS + GCS + GRS had AUC $=0.810$, GLS + GCS had AUC $=0.810$, GCS + GRS had AUC =0.802, and GLS + GRS had AUC =0.787, indicating that GRS had no significant predictive value here.

Table 3 Comparison of parameters between the MVA group and the non-MVA group

\begin{tabular}{lccc}
\hline Variable & MVA $(\mathrm{n}=54)$ & Non-MVA $(\mathrm{n}=107)$ & P value \\
\hline Clinical parameters & & & $<0.001^{*}$ \\
BNP $(\mathrm{pg} / \mathrm{mL})$ & $2,152.9(1,843.8-2,568.0)$ & $837.1(148.3-667.6)$ & $<0.001^{*}$ \\
$\mathrm{~T}_{\mathrm{p}} \mathrm{T}_{\mathrm{e}}(\mathrm{ms})$ & $103.3(96.7-119.2)$ & & $<0.0-90.0)$ \\
CMR parameters & & $30.0(26.0-34.0)$ & $<0.001^{*}$ \\
LVEF $(\%)$ & $23.0(20.0-26.0)$ & $-10.2(-8.5$ to -11.8$)$ & $<0.001^{*}$ \\
GLS $(\%)$ & $-6.6(-5.2$ to -8.3$)$ & $-10.3(-8.5$ to -12.2$)$ & $<0.001^{*}$ \\
GCS $(\%)$ & $-6.7(-5.2$ to -8.3$)$ & $12.3(8.3-15.5)$ & \\
GRS $(\%)$ & $6.8(4.7-10.0)$ & & \\
\hline
\end{tabular}

Data are displayed as medians, with interquartile ranges in parentheses. *, statistically significant difference. MVA, malignant ventricular arrhythmia; BNP, brain natriuretic peptide; LVEF, left ventricular ejection fraction; $T_{p}-T_{e}, T_{p e a k}-T_{\text {end }}$ interval; GLS, global longitudinal strain; GCS, global circumferential strain; GRS, global radial strain.

Table 4 ROC analysis curve of BNP, LVEF, $T_{p}-T_{e}$, GLS, GCS, and GRS in the MVA group

\begin{tabular}{|c|c|c|c|c|c|}
\hline Variable & Cut-off & AUC & $95 \% \mathrm{Cl}$ & Sensitivity & Specificity \\
\hline BNP $(\mathrm{pg} / \mathrm{mL})$ & 1,294 & 0.959 & $0.931-0.987$ & 0.889 & 0.888 \\
\hline $\mathrm{T}_{\mathrm{p}}-\mathrm{T}_{\mathrm{e}}(\mathrm{ms})$ & 96 & 0.859 & $0.797-0.921$ & 0.804 & 0.791 \\
\hline \multicolumn{6}{|c|}{ CMR parameters } \\
\hline GLS (\%) & -8.375 & 0.795 & $0.725-0.866$ & 0.778 & 0.757 \\
\hline GCS (\%) & -8.305 & 0.802 & $0.731-0.872$ & 0.796 & 0.776 \\
\hline GRS (\%) & 9.105 & 0.754 & $0.676-0.832$ & 0.741 & 0.692 \\
\hline
\end{tabular}

ROC, receiver operating characteristic; MVA, malignant ventricular arrhythmia; BNP, brain natriuretic peptide; LVEF, left ventricular ejection fraction; $T_{p}-T_{e}$, Tpeak-Tend interval; GLS, global longitudinal strain; GCS, global circumferential strain; GRS, global radial strain; AUC, area under the receiver operating characteristic curve; $\mathrm{Cl}$, confidence interval. 
A

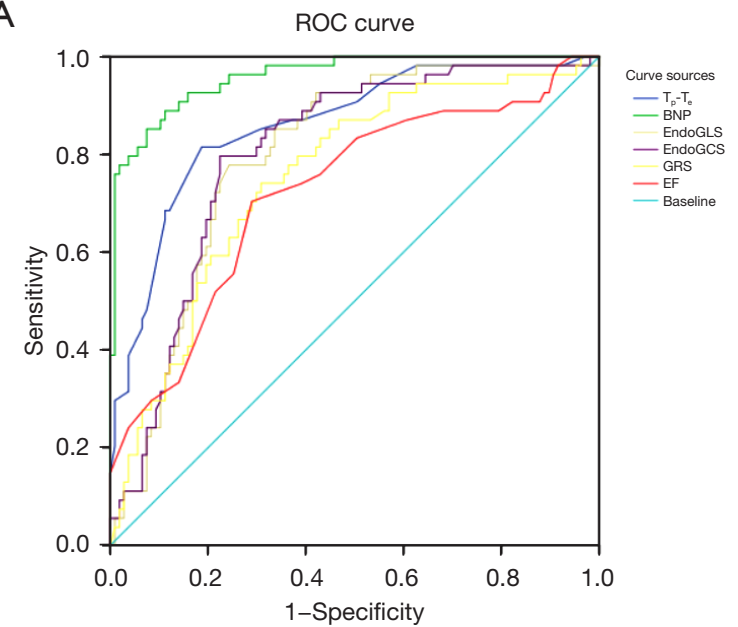

B

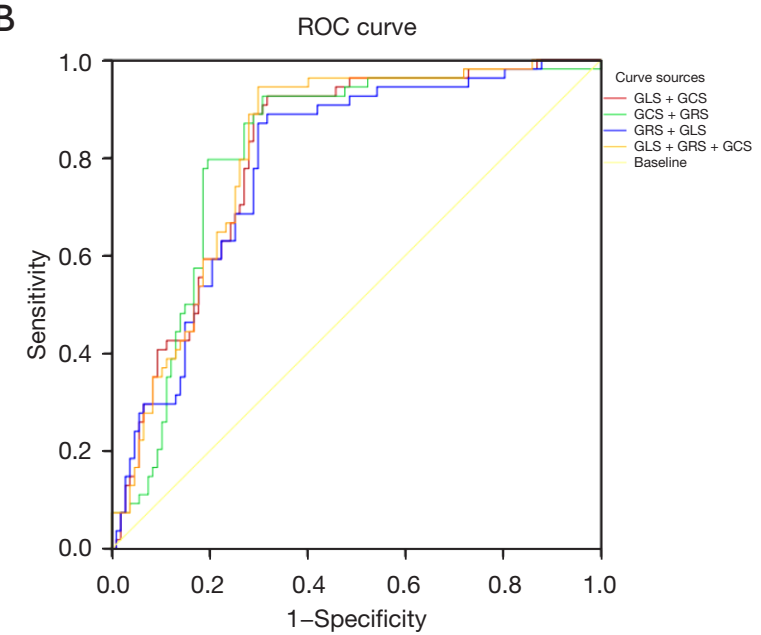

Figure 3 (A) ROC curves for predicting MVA in NIDCM patients: BNP: AUC $=0.959$; $T_{\mathrm{p}}-\mathrm{T}_{\mathrm{e}}$ : $\mathrm{AUC}=0.859$; LVEF: AUC $=0.672$; GLS: $\mathrm{AUC}=0.795$; GCS: $\mathrm{AUC}=0.802$; GRS: AUC $=0.754$. (B) ROC curves for predicting MVA in NIDCM patients: GLS + GCS + GRS: AUC $=0.810 ;$ GLS + GCS: AUC $=0.810 ;$ GCS + GRS: $\mathrm{AUC}=0.802 ;$ GLS + GRS: AUC $=0.787$. The yellow line (GLS + GCS + GRS) coincides with the green line (GLS + GCS). ROC, receiver operating characteristic; MVA, malignant ventricular arrhythmia; NIDCM, nonischemic dilated cardiomyopathy; BNP, brain natriuretic peptide; $T_{p}-T_{e}, T_{\text {peak }}-T_{\text {end }}$ interval; LVEF, left ventricular ejection fraction; GLS, global longitudinal strain; GCS, global circumferential strain; GRS, global radial strain.

Table 5 ROC analysis curve of CMR-FT characteristics of patients with MVA

\begin{tabular}{llccc}
\hline Variable & AUC & $95 \% \mathrm{Cl}$ & Sensitivity & Specificity \\
\hline GLS & 0.795 & $0.725-0.866$ & 0.778 & 0.757 \\
GCS & 0.802 & $0.731-0.872$ & 0.796 & 0.776 \\
GRS & 0.754 & $0.676-0.832$ & 0.741 & 0.625 \\
GLS+GCS+GRS & 0.810 & $0.742-0.877$ & 0.944 & 0.701 \\
GLS+GCS & 0.810 & $0.742-0.877$ & 0.926 & 0.682 \\
GCS+GRS & 0.802 & $0.731-0.872$ & 0.926 & 0.692 \\
GLS+GRS & 0.787 & $0.715-0.859$ & 0.889 & 0.682 \\
\hline
\end{tabular}

ROC, receiver operating characteristic; MVA, malignant ventricular arrhythmia; GLS, global longitudinal strain; GCS, global circumferential strain; GRS, global radial strain; AUC, area under the receiver operating characteristic curve; CMR-FT, cardiac magnetic resonance feature tracking; $\mathrm{Cl}$, confidence interval.

When GCS and GLS were selected to jointly predict the occurrence of MVA, the AUC and prediction probability $\mathrm{P}$ were higher than those of predictions based on each single indicator (Table 5, Figure 3B).

\section{Discussion}

The main findings of this study were that $3 \mathrm{D}$ myocardial strain parameters (GLS, GCS, and GRS) measured by CMR-FT had certain clinical value in reflecting the underlying abnormality of ventricular mechanics of NIDCM with MVA. In particular, GCS and GLS had higher diagnostic efficiency, sensitivity, and specificity compared with other magnetic resonance parameters. CMR-FT had good interobserver agreement and thus could be used as an effective clinical evaluation method, which is also consistent with other study results $(15,16)$.

In nonischemic cardiomyopathy, myocardial fibrosis is most likely to involve the left ventricular subintima or the whole cardiomyocyte intercellular substance or perivascular 
tissue (17), showing a diffuse distribution. Excessive fibrosis plays an important role in ventricular structural remodeling and electrical remodeling. The interlacing of survival cardiomyocytes and collagen fibers promotes the propagation and development of reentrant ventricular tachycardia (18). The pathogenesis of sudden cardiac death in NIDCM patients is mainly caused by persistent ventricular tachycardia and fibrillation. The clinical guidelines for the treatment and management of such patients are mostly based on LVEF. One study has suggested that LVEF is an important prognostic factor in NIDCM and recommended an implantable cardioverter-defibrillator for patients with heart failure whose LVEF is less than 35\% (19). In 2010, the American Heart Association (Expert Consensus on Cardiovascular Magnetic Resonance) proposed that cardiac magnetic resonance could be used as a gold standard for assessing cardiac structure and function. Many authors also believe that cardiac magnetic resonance is more accurate and reliable than echocardiography in evaluating cardiac function $(20,21)$. However, in clinical practice, sudden cardiac death can occur in patients with normal or mildly decreased ejection fraction. Therefore, some researchers believe that the diagnostic value of LVEF for sudden cardiac death risk is relatively low $(22,23)$, which is also consistent with the findings of this study (LVEF, AUC $=0.672)$. It is believed that the subtle abnormal changes of cardiac function can be found earlier by the myocardial strain technique than by measuring $\operatorname{LVEG}(7,8)$. The principle of the more frequently used ultrasonic speckle tracking is similar to that of CMR-FT, but ultrasonic speckle tracking is limited by a low spatial resolution of ultrasonic imaging, poor sound window, and operator skills and needs real-time processing. Therefore, the accuracy and convenience of myocardial strain measurement still need to be improved (24). The myocardial strain parameters measured by CMR-FT have good consistency with ultrasonic speckle tracking and magnetic resonance tagging. CMR-FT is expected to become a new reliable technique for measuring myocardial strain that may replace ultrasonic speckle tracking and magnetic resonance tagging $(25,26)$.

There are few studies on the performance of CMR-FT in predicting ventricular arrhythmia in NIDCM. One study has shown that left ventricular systolic circumferential strain rate is an independent predictor of ventricular tachycardia in patients with tetralogy of Fallot (27). GLS represents the shortening of cardiac muscle fibers from the basal segment to the apical segment in the long-axis direction, which is expressed as a negative value; GCS represents the change of cardiac muscle fiber shortening inwardly along the circumferential direction in the short-axis direction, which is expressed as a negative value; GRS represents the deformation of cardiac muscle fibers toward the center of the heart cavity, reflecting the change in thickness of cardiac muscle fibers in the center of the heart cycle, which is expressed as a positive value. Our results showed although GLS (AUC $=0.795)$, GCS (AUC $=0.802$ ), and GRS (AUC $=0.754)$ were not as effective as the conventionally used $\mathrm{BNP}(\mathrm{AUC}=0.959)$ and $\mathrm{T}_{\mathrm{p}}-\mathrm{T}_{\mathrm{e}}(\mathrm{AUC}=0.859)$ in predicting the occurrence of MVA in NIDCM, they were more effective than LVEF (AUC =0.672), giving them certain diagnostic value. Among them, the diagnostic efficiency of GCS (AUC $=0.802$ ) was similar to that of $T_{p}-T_{e}$ (AUC $=0.859)$. GCS had high sensitivity and specificity ( 0.796 and 0.776 , respectively), in line with previous findings that left ventricular myocardial GCS was an independent predictor of adverse events in NIDCM patients (28). Although the diagnostic efficiency of GLS (AUC $=0.795$ ) was slightly lower than that of GCS (AUC $=0.802$ ), GLS has been associated with poor prognosis in NIDCM patients $(29,30)$. Single-factor ROC analysis showed that GCS and GLS had high diagnostic efficiency in predicting the occurrence of MVA in NIDCM. Multifactor ROC analysis showed that the efficiency of diagnosis predicted from the combination of GCS and GLS (AUC $=0.810$ ) was better than that predicted from each single indicator. The diagnostic efficiency of GRS was relatively limited, indicating that myocardial fibrosis in patients with NIDCM and MVA in this study might be mainly involved in the circumferential and longitudinal directions, which was also basically consistent with the enhanced mode of myocardial fibrosis in NIDCM patients on LGE images in clinical practice. This may further suggest that the occurrence of MVA may be related to the diversity and complexity of the myocardial fibrosis involved. Erley et al. have suggested that GCS is more likely to reflect the presence of myocardial LGE than GLS is (26). We found that the cut-off values of GCS and GLS were $-8.305 \%$ and $-8.375 \%$, respectively, indicating that the overall myocardial strain of NIDCM patients with MVA had basically the same impairment level in the longitudinal and radial directions. However, accurate cut-off values for them are still lacking and need to be confirmed by larger, multicenter studies. GCS and GLS may be good indicators for independently predicting the occurrence of MVA in NIDCM patients in the future, but there is no study on whether GCS and GLS can provide a reference for clinical treatment with an implantable cardioverter- 
defibrillator. In addition, compared with LGE and T1 mapping, CMR-FT can evaluate ventricular arrhythmia without any contrast agent, which is important for patients with contrast agent contraindications.

In our study, each cardiac cine sequence consisted of 20 25 frames of images per cardiac cycle. Cardiac cine images with low temporal resolution can easily cause a loss of real cardiac motion information. Past research has suggested that the CMR-FT cardiac cine sequence composed of 20-30 frames of images per cardiac cycle underestimates the real myocardial strain (31). Still, previous studies that used cardiac cine sequences composed of 20-30 frames of images per cardiac cycle have shown that such cardiac cine sequences can provide useful information for clinical practice (32-34).

This study has many shortcomings. First, it was a small single-center study, so the results cannot be universally generalized. Larger, prospective multicenter studies are needed to confirm its results. Second, this was a retrospective study, some patients could receive antiarrhythmia treatment during this period. Although data collection and study methods were carefully selected, there might be some errors in the results. For example, the cutoff value of $\mathrm{T}_{\mathrm{p}}-\mathrm{T}_{\mathrm{e}}$ in this study was $96 \mathrm{~ms}$. Tse $e t$ al. have suggested that the cut-off value of $\mathrm{T}_{\mathrm{p}}-\mathrm{T}_{\mathrm{e}}$ should be $103.3 \mathrm{~ms}$ in their meta-analysis (35). Third, some NIDCM patients may have arrhythmia during CMR examination, and the reliability of cardiac cine image remains to be further studied. Fourth, preliminarily explore the value of cardiac magnetic resonance feature tracking (CMR-FT) for MVA in dilated cardiomyopathy. It did not follow up the NIDCM patients, record the occurrence of adverse cardiovascular events, or assess the relationship between left ventricular strain and the long-term prognosis of NIDCM patients.

\section{Conclusions}

In general, the 3D myocardial strain parameters (GLS, GCS, and GRS) measured by CMR-FT had certain diagnostic value and could reflect the underlying abnormality of ventricular mechanics of NIDCM with MVA. In particular, GCS and GLS had higher diagnostic efficiency than LVEF, which provides a new direction for the research in this field. However, the prognostic value of myocardial strain for NIDCM with MVA needs further follow-up studies.

\section{Acknowledgments}

The authors appreciate the academic support from the
AME Radiology Collaborative Group.

Funding: This work was supported by the grant from Sichuan Science and Technology Program (No. 2020YFS0558) to SK Peng, and was also supported in part by the Foundation for Young Scientists of Sichuan provincial people's hospital (No. 2021QN08) to SK Peng.

\section{Footnote}

Reporting Checklist: The authors have completed the STARD reporting checklist. Available at https://atm.amegroups. com/article/view/10.21037/atm-22-660/rc

Data Sharing Statement: Available at https://atm.amegroups. com/article/view/10.21037/atm-22-660/dss

Conflicts of Interest: All authors have completed the ICMJE uniform disclosure form (available at https://atm. amegroups.com/article/view/10.21037/atm-22-660/coif). The authors have no conflicts of interest to declare.

Ethical Statement: The authors are accountable for all aspects of the work in ensuring that questions related to the accuracy or integrity of any part of the work are appropriately investigated and resolved. All procedures performed in this study involving human participants were in accordance with the Declaration of Helsinki (as revised in 2013). The study was approved by the Institutional Ethics Review Committee of the Sichuan Provincial People's Hospital (No. 538). Individual consent for this retrospective analysis was waived.

Open Access Statement: This is an Open Access article distributed in accordance with the Creative Commons Attribution-NonCommercial-NoDerivs 4.0 International License (CC BY-NC-ND 4.0), which permits the noncommercial replication and distribution of the article with the strict proviso that no changes or edits are made and the original work is properly cited (including links to both the formal publication through the relevant DOI and the license). See: https://creativecommons.org/licenses/by-nc-nd/4.0/.

\section{References}

1. Halliday BP, Cleland JGF, Goldberger JJ, et al. Personalizing Risk Stratification for Sudden Death in Dilated Cardiomyopathy: The Past, Present, and Future. Circulation 2017;136:215-31. 
2. Levine YC, Rosenberg MA, Mittleman M, et al. B-type natriuretic peptide is a major predictor of ventricular tachyarrhythmias. Heart Rhythm 2014;11:1109-16.

3. Antzelevitch C, Di Diego JM. Tpeak-Tend interval as a marker of arrhythmic risk. Heart Rhythm 2019;16:954-5.

4. Alba AC, Gaztañaga J, Foroutan F, et al. Prognostic Value of Late Gadolinium Enhancement for the Prediction of Cardiovascular Outcomes in Dilated Cardiomyopathy: An International, Multi-Institutional Study of the MINICOR Group. Circ Cardiovasc Imaging 2020;13:e010105.

5. Li S, Zhou D, Sirajuddin A, et al. T1 Mapping and Extracellular Volume Fraction in Dilated Cardiomyopathy: A Prognosis Study. JACC Cardiovasc Imaging 2021. [Epub ahead of print].

6. Jeung MY, Germain P, Croisille P, et al. Myocardial tagging with MR imaging: overview of normal and pathologic findings. Radiographics 2012;32:1381-98.

7. Scatteia A, Baritussio A, Bucciarelli-Ducci C. Strain imaging using cardiac magnetic resonance. Heart Fail Rev 2017;22:465-76.

8. Cui Y, Cao Y, Song J, et al. Association between myocardial extracellular volume and strain analysis through cardiovascular magnetic resonance with histological myocardial fibrosis in patients awaiting heart transplantation. J Cardiovasc Magn Reson 2018;20:25.

9. Gatti M, Palmisano A, Faletti R, et al. Two-dimensional and three-dimensional cardiac magnetic resonance featuretracking myocardial strain analysis in acute myocarditis patients with preserved ejection fraction. Int $\mathrm{J}$ Cardiovasc Imaging 2019;35:1101-9.

10. Liu T, Gao Y, Wang H, et al. Association between right ventricular strain and outcomes in patients with dilated cardiomyopathy. Heart 2020. [Epub ahead of print].

11. Park SM, Kim YH, Ahn CM, et al. Relationship between ultrasonic tissue characterization and myocardial deformation for prediction of left ventricular reverse remodelling in non-ischaemic dilated cardiomyopathy. Eur J Echocardiogr 2011;12:887-94.

12. Maron BJ, Towbin JA, Thiene G, et al. Contemporary definitions and classification of the cardiomyopathy: an American heart association scientific statement from the councilon clinical cardiology, heart failure and transplantation committee; quality of care and outcomes research and functional genomics and translational biology interdisciplinary working groups; and council on epidemiology and prevention. Circulation 2006;113:1807-16

13. He J, Yang $W, W u W$, et al. Early Diastolic Longitudinal
Strain Rate at MRI and Outcomes in Heart Failure with Preserved Ejection Fraction. Radiology 2021;301:582-92.

14. He J, Sirajuddin A, Li S, et al. Heart Failure With Preserved Ejection Fraction in Hypertension Patients: A Myocardial MR Strain Study. J Magn Reson Imaging 2021;53:527-39.

15. Truong VT, Palmer C, Wolking S, et al. Normal left atrial strain and strain rate using cardiac magnetic resonance feature tracking in healthy volunteers. Eur Heart J Cardiovasc Imaging 2020;21:446-53.

16. Schuster A, Stahnke VC, Unterberg-Buchwald C, et al. Cardiovascular magnetic resonance feature-tracking assessment of myocardial mechanics: Intervendor agreement and considerations regarding reproducibility. Clin Radiol 2015;70:989-98.

17. Hare JM, Walford GD, Hruban RH, et al. Ischemic cardiomyopathy: endomyocardial biopsy and ventriculographic evaluation of patients with congestive heart failure, dilated cardiomyopathy and coronary artery disease. J Am Coll Cardiol 1992;20:1318-25.

18. Morita N, Mandel WJ, Kobayashi Y, et al. Cardiac fibrosis as a determinant of ventricular tachyarrhythmias. J Arrhythm 2014;30:389-94.

19. Pradella S, Grazzini G, De Amicis C, et al. Cardiac magnetic resonance in hypertrophic and dilated cardiomyopathies. Radiol Med 2020;125:1056-71.

20. Champ-Rigot L, Gay P, Seita F, et al. Clinical outcomes after primary prevention defibrillator implantation are better predicted when the left ventricular ejection fraction is assessed by cardiovascular magnetic resonance. $\mathrm{J}$ Cardiovasc Magn Reson 2020;22:48.

21. Kinno M, Nagpal P, Horgan S, et al. Comparison of Echocardiography, Cardiac Magnetic Resonance, and Computed Tomographic Imaging for the Evaluation of Left Ventricular Myocardial Function: Part 1 (Global Assessment). Curr Cardiol Rep 2017;19:9.

22. Køber L, Thune JJ, Nielsen JC, et al. Defibrillator Implantation in Patients with Nonischemic Systolic Heart Failure. N Engl J Med 2016;375:1221-30.

23. Centurión OA, Alderete JF, Torales JM, et al. Myocardial Fibrosis as a Pathway of Prediction of Ventricular Arrhythmias and Sudden Cardiac Death in Patients With Nonischemic Dilated Cardiomyopathy. Crit Pathw Cardiol 2019;18:89-97.

24. Claus P, Omar AMS, Pedrizzetti G, et al. Tissue Tracking Technology for Assessing Cardiac Mechanics: Principles, Normal Values, and Clinical Applications. JACC Cardiovasc Imaging 2015;8:1444-60. 
25. van Everdingen WM, Zweerink A, Nijveldt R, et al. Comparison of strain imaging techniques in CRT candidates: CMR tagging, CMR feature tracking and speckle tracking echocardiography. Int J Cardiovasc Imaging 2018;34:443-56.

26. Erley J, Genovese D, Tapaskar N, et al. Echocardiography and cardiovascular magnetic resonance based evaluation of myocardial strain and relationship with late gadolinium enhancement. J Cardiovasc Magn Reson 2019;21:46.

27. Hagdorn QAJ, Vos JDL, Beurskens NEG, et al. CMR feature tracking left ventricular strain-rate predicts ventricular tachyarrhythmia, but not deterioration of ventricular function in patients with repaired tetralogy of Fallot. Int J Cardiol 2019;295:1-6.

28. Shu SL, Wang J, Wang C, et al. Prognostic Value of Feature-Tracking Circumferential Strain in Dilated Cardiomyopathy Patients with Severely Reduced Ejection Fraction Incremental to Late Gadolinium Enhancement. Curr Med Sci 2021;41:158-66.

29. Kammerlander AA, Donà C, Nitsche C, et al. Feature Tracking of Global Longitudinal Strain by Using Cardiovascular MRI Improves Risk Stratification in Heart Failure with Preserved Ejection Fraction. Radiology 2020;296:290-8.

30. Romano S, Judd RM, Kim RJ, et al. Feature-Tracking Global Longitudinal Strain Predicts Mortality in Patients

Cite this article as: Song L, Zhao X, Lv W, Zeng J, Wang Y, Gong B, Kalogeropoulos AP, Pu H, Bai Y, Peng S. Preliminary study on the diagnostic value of cardiac magnetic resonance feature tracking for malignant ventricular arrhythmias in non-ischemic dilated cardiomyopathy. Ann Transl Med 2022;10(4):215. doi: 10.21037/atm-22-660
With Preserved Ejection Fraction: A Multicenter Study. JACC Cardiovasc Imaging 2020;13:940-7.

31. Amzulescu MS, De Craene M, Langet H, et al. Myocardial strain imaging: review of general principles, validation, and sources of discrepancies. Eur Heart J Cardiovasc Imaging 2019;20:605-19.

32. Wu L, Germans T, Güçlü A, et al. Feature tracking compared with tissue tagging measurements of segmental strain by cardiovascular magnetic resonance. J Cardiovasc Magn Reson 2014;16:10.

33. von Roeder M, Rommel KP, Kowallick JT, et al. Influence of Left Atrial Function on Exercise Capacity and Left Ventricular Function in Patients With Heart Failure and Preserved Ejection Fraction. Circ Cardiovasc Imaging 2017;10:e005467.

34. Ito $\mathrm{H}$, Ishida $\mathrm{M}$, Makino $\mathrm{W}$, et al. Cardiovascular magnetic resonance feature tracking for characterization of patients with heart failure with preserved ejection fraction: correlation of global longitudinal strain with invasive diastolic functional indices. J Cardiovasc Magn Reson 2020;22:42.

35. Tse G, Gong M, Wong WT, et al. The Tpeak - Tend interval as an electrocardiographic risk marker of arrhythmic and mortality outcomes: A systematic review and metaanalysis. Heart Rhythm 2017;14:1131-7. 